\title{
Lensless x-ray imaging in reflection geometry
}

\author{
S. Roy ${ }^{1, *}$ D. Parks,,${ }^{1,2}$ K. A. Seu, ${ }^{1,2}$ R. Su, ${ }^{1,2}$ J. J. Turner, ${ }^{3}$ \\ W. Chao, ${ }^{4}$ E. H. Anderson, ${ }^{4}$ S. Cabrini, ${ }^{5}$ and S. D. Kevan ${ }^{2}$ \\ ${ }^{1}$ Advanced Light Source, Lawrence Berkeley National Laboratory, Berkeley, California 94720, USA \\ ${ }^{2}$ Department of Physics, University of Oregon, Eugene, Oregon 97403, USA \\ ${ }^{3}$ SLAC National Accelerator Laboratory, Menlo Park, California 94025, USA \\ ${ }^{4}$ Center for X-ray Optics, Lawrence Berkeley National \\ Laboratory, Berkeley, California 94720, USA \\ ${ }^{5}$ Molecular Foundry, Lawrence Berkeley National Laboratory, Berkeley, California 94720, USA
}

(Dated: January 5, 2011) 


\section{DISCLAIMER}

This document was prepared as an account of work sponsored by the United States Government. While this document is believed to contain correct information, neither the United States Government nor any agency thereof, nor The Regents of the University of California, nor any of their employees, makes any warranty, express or implied, or assumes any legal responsibility for the accuracy, completeness, or usefulness of any information, apparatus, product, or process disclosed, or represents that its use would not infringe privately owned rights. Reference herein to any specific commercial product, process, or service by its trade name, trademark, manufacturer, or otherwise, does not necessarily constitute or imply its endorsement, recommendation, or favoring by the United States Government or any agency thereof, or The Regents of the University of California. The views and opinions of authors expressed herein do not necessarily state or reflect those of the United States Government or any agency thereof or The Regents of the University of California. 
Lensless x-ray imaging techniques such as coherent diffraction imaging $(\mathrm{CDI})^{1-8}$ and ptychography ${ }^{9-11}$, and Fourier transform holography $(\mathrm{FTH})^{12-17}$ can provide time-resolved, diffraction-limited images. Nearly all examples of these techniques have been focused on transmission geometry, restricting the samples and reciprocal spaces which can be investigated. We report a lensless x-ray technique developed for imaging in Bragg and small-angle scattering geometries which may also see application in transmission geometries. We demonstrate this by imaging a nanofabricated pseudorandom binary structure in small angle reflection geometry. The technique can be used with extended objects, places no restriction on sample size, and requires no additional sample masking. The realization of x-ray lensless imaging in reflection geometry opens up the possibility of single-shot imaging of surfaces in thin films, buried interfaces in magnetic multilayers, organic photovoltaic and field effect transistor devices, or Bragg planes in a single crystal.

In this method, an exit screen containing several holes acts as a phase encoder to define the object and reference waves after the exit wave has already propagated some distance away from the sample ${ }^{18-20}$. In the limit of point reference holes, this forms a Fourier transform hologram of the wave at the plane of the screen. When larger references are required to overcome low photon flux, we use iterative techniques from CDI to recover the wave at the screen. Limiting the field of view after scattering allows a support constraint on an extended sample without modifying the sample itself, eliminating the need for special sample fabrication and potentially leading to high-throughput imaging. The ability to operate in reflection makes it possible to use non-transmissive samples and choose a reciprocal q-value corresponding to an electronic or magnetic surface, interface or lattice feature.

Figure 1(a) shows the experimental geometry. The partially coherent x-ray beam is incident on a sample at a certain angle $\theta$. The beam scatters from the sample and passes through an exit screen, which is placed a distance $z$ from the sample. The screen splits the scattered signal and creates an object and reference which interfere in the far field at a detector centered at the corresponding $2 \theta$ angle. The separation of the exit screen from the sample surface leads to a two step solution procedure for calculating the wave leaving the sample. First, an estimate of the wave leaving the exit screen is obtained either by a Fourier transform in the FTH limit or by iterative phase retrieval methods otherwise. The 
convergence of iterative methods is improved by the presence of the reference hole ${ }^{21}$. Second, the recovered complex wave at the exit screen plane is then back-propagated to the sample surface. Minimizing the distance between the screen and the sample is critically important as two effects limit the effective range of back-propagation: the limited numerical aperture of the large hole in the exit screen leads it to act as a a low-pass filter on the diffracted wave and the Fresnel zones arising from back-propagating the circular aperture may overwhelm the recovered exit wave at the sample if the propagation distance is too great.

To determine an effective working limit for the distance separating the sample and the exit screen we performed numerical simulations of the scattering from several representative aperiodic structures. In these simulations, an image of the sample was propagated to the exit screen by convolution with the Fresnel propagator, then to the detector by Fourier transform. These simulations confirm the necessity of back-propagating the wave recovered at the screen, as near-field diffraction patterns arising from nano-scale features are recognizable only at very close distances which cannot be reasonably achieved in our reflection geometry. We estimate that the ringing from back propagating the object hole becomes problematic for a Fresnel number of approximately 5. For a pinhole of $2 \mu \mathrm{m}$ diameter at $500 \mathrm{eV}$, this corresponds to a propagation limit of about $100 \mu \mathrm{m}$.

Figure 2(a) shows the data obtained due to scattering from a nanostructured pseudorandom binary structure shown in Fig. 1(c). The data display speckle pattern characteristic of coherent x-ray scattering and the orthogonal fringing of the speckle is due to the two reference waves. The maximum recordable momentum transfer was $\mathbf{q}=0.198 \mathrm{~nm}^{-1}$, which upon inverting the far field image gives a theoretically minimum resolvable feature size of $32 \mathrm{~nm}$. The phase-retrieval transfer function of the reconstruction indicates that minimum resolvable feature size is approximately $60 \mathrm{~nm}$. Figure 2(b) shows a two dimensional Fourier transform of the data, the components of which are the auto- and cross-correlations between the object and reference holes as expected from their positions on the exit screen. Phase reconstruction was achieved by the Hybrid Input-Output and Error Reduction algorithms ${ }^{22}$ and Marchesini's Shrinkwrap algorithm ${ }^{23}$. The magnitude component of the phase retrieved image at the screen is shown in Fig. 2(c). We have not corrected for the partial coherent nature of the incident x-ray beam. The aperture array at the exit screen was designed to fit within the transverse coherence length of the beam and the field within the object aperture can be assumed fully coherent. We believe that the primary effect of the partial coherence 
is a reduction in contrast of the fringes from interference between the object and horizontal reference apertures, which manifests as the lower intensity of the reconstructed horizontal reference compared to the vertical reference.

After reconstructing the wave at the exit screen we back-propagated the solution through a range of distances, several of which are shown in Fig. 3(a-d); the sample-screen separation distance was initially estimated ex situ by visible-light microscopy as $80 \mu \mathrm{m}$. To quantitatively determine the correct propagation distance we calculated the acutance ${ }^{24}$ (a measure of edge sharpness) of the complex propagated field, as we expect the correctly propagated field to display the sharpest features. The acutance of the wave field is defined as the integral of the square of the spatial gradient of the magnitude component of the propagated field. This metric is shown for forward and backward directions in Figure 4. The qualitative behavior of the acutance metric (high at the exit screen plane decreasing upon propagation, with a peak at the sample) is consistent with our simulations. In particular, simulations show a general decrease in acutance upon propagation from the exit screen plane when the aperture is not significantly larger than the features of the sample. The peak position at $97 \mu \mathrm{m}$ corresponds to the back-propagation distance for the image to be on the sample plane where the features of the test pattern are in best focus.

Visual inspection of the back-propagation sequence also estimates the separation distance to be approximately $97 \mu \mathrm{m}$. At this distance, excellent agreement is seen between the backpropagated wave (Fig. 3 (c)) and the test pattern after accounting for the geometrical effects of the surface features along the beam direction due to the shallow angle of incidence (Fig. 3 (e)). In comparison, Figure 3 (f) shows the back-propagation of the cross-correlation term obtained by a single Fourier transform of the hologram without improvement by iterative phasing algorithms. The largest features of the test pattern are clearly discernible, but the image is poorly resolved due to large references needed to compensate for low photon flux as well as structure within the references. This underscores the necessity of using iterative phase retrieval methods to complement non-ideal references.

Even at optimal focus, the incident beam is approximately flat over the object aperture's field of view and no explicit consideration of beam profile is necessary when interpreting the outgoing wave. The contrast between bright and dark regions of the shown magnitude component demonstrate the difference in reflectivity between the deposited nickel and the silicon substrate. 
In conclusion, we have successfully demonstrated a method of lensless x-ray imaging in reflection by phase encoding the exit wave. It can use an extended sample as in a conventional scattering experiment without the need for special sample preparation, and can give an image in a single shot thereby providing the framework to perform high throughput imaging experiments. The technique is versatile and is applicable to a large range of reciprocal qvalues. Furthermore, measurement as a function of incident and exit angles allows extension of the technique to 3D reconstruction. The resolution of the recovered image is limited in part by the reference size, which is presently constrained by photon intensity and may be improved considerably by x-ray FEL sources. Resolution and the image quality can also be improved by increasing the numerical aperture of the object hole, which is currently limited by the transverse coherence length of the incident beam. Combining resonant $\mathrm{x}-$ ray scattering with imaging in reflection provides for a very powerful technique that we hope could be readily and routinely applied to study a broad range of surface and interface features of devices and nano-structures in hard and soft condensed matter which have not yet been imaged.

\section{METHOD}

The exit screen in Fig. 1(b) was fabricated on a SiN membrane coated with $1 \mu \mathrm{m}$ of $\mathrm{Au}$ using a focused ion beam to create the circular apertures. The diameter of the holes is 2 $\mu \mathrm{m}$ for the large hole and $0.64 \mu \mathrm{m}$ for the small holes. The test pattern in Fig. 1(c) is a pseudo-random nickel on silicon nano-structure created with electron beam lithography. The experiment was performed at beamline 12.0.2.2 of the Advanced Light Source, Lawrence Berkeley National Laboratory. X-ray beam of $500 \mathrm{eV}$ energy are incident at an angle of 17 degrees and the reflected light is recorded on a charge coupled device $0.350 \mathrm{~m}$ away. 60 acquisitions totaling 180 minutes of time were used; the same analysis would have been successful with an order of magnitude less integration time. Phase reconstruction alternated 19 iterations of the hybrid input-output algorithm and 1 iteration of the error-reduction algorithm ${ }^{22}$ for several hundred total iterations. The initial support for the reconstruction was supplied by the outlines and positions of the cross-correlation terms in the inversion of the far-field intensity, and was periodically updated using the Shrinkwrap algorithm ${ }^{23}$. The final result is an average of reconstructions from several random initial seeds; using 
the inversion of the far-field intensity with small random perturbations did not yield an improved reconstruction. Back propagation to the sample plane was performed by numerical convolution with the Fresnel propagator. The acutance calculated at each distance is here defined as the integral of the square of the gradient of the magnitude component of the propagated field, with the gradient approximated by the quadrature sum of two Sobel filters and the region of integration a circle of radius 30 pixels co-centered with the reconstructed object aperture.

\section{AUTHOR CONTRIBUTIONS}

S. Roy conceived the experiment. S. Roy, K. A. Seu and D. Parks designed the experiment. W. Chao and E. H. Anderson fabricated the test pattern. S. Cabrini fabricated the exit screen. D. Parks, K. A. Seu, R. Su, and S. Roy preformed the experiments, and D. Parks, K. A. Seu and S. Roy analyzed data. S. Roy, K. A. Seu, D. Parks, J. J. Turner, and S. D. Kevan authored the paper. All authors discussed the result and contributed to the final manuscript.

\section{COMPETING FINANCIAL INTERESTS}

The authors declare no competing financial interests.

\section{REFERENCES}

* Correspondence and requests for materials should be addressed to:SRoy@lbl.gov

1 Nugent, K. A. Coherent methods in the x-ray sciences. Advances in Physics 59, 1 - 99 (2010).

2 Miao, J., Charalambous, P., Kirz, J. \& Sayre, D. Extending the methodology of x-ray crystallography to allow imaging of micrometre-sized non-crystalline specimens. Nature 400, 342-344 (1999).

3 Chapman, H. N. et al. Femtosecond time-delay x-ray holography. Nature 448, 676-679 (2007). 
4 Chapman, H. N. et al. High-resolution ab initio three-dimensional x-ray diffraction microscopy. J. Opt. Soc. Am. A 23, 1179-1200 (2006).

5 Nugent, K. A., Peele, A. G., Chapman, H. N. \& Mancuso, A. P. Unique phase recovery for nonperiodic objects. Phys. Rev. Lett. 91, 203902 (2003).

6 Shapiro, D. et al. Biological imaging by soft x-ray diffraction microscopy. Proceedings of the National Academy of Sciences 102, 15343-15346 (2005).

7 Nelson, J. et al. High-resolution x-ray diffraction microscopy of specifically labeled yeast cells. Proceedings of the National Academy of Sciences 107, 7235-7239 (2010).

8 Pfeifer, M. A., Williams, G. J., Vartanyants, I. A., Harder, R. \& Robinson, I. K. Threedimensional mapping of a deformation field inside a nanocrystal. Nature 442, 63-66 (2006).

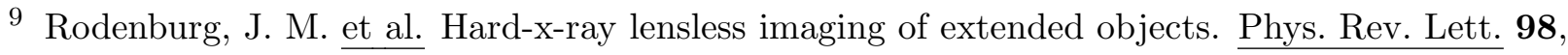
034801 (2007).

10 Thibault, P. et al. High-ResolutionScanning X-ray Diffraction Microscopy. Science 321, 379-382 (2008).

11 Dierolf, M. et al. Ptychographic coherent diffractive imaging of weakly scattering specimens. New Journal of Physics 12, 035017 (2010).

12 McNulty, I. et al. High-Resolution Imaging by Fourier Transform X-ray Holography. Science 256, 1009-1012 (1992).

13 Marchesini, S. et al. Massively parallel x-ray holography. Nature Photonics 2, 560-563 (2008).

14 Eisebitt, S. et al. Lensless imaging of magnetic nanostructures by x-ray spectro-holography. Nature 432, 885-888 (2004).

15 Chamard, V. et al. Three-dimensional x-ray fourier transform holography: The bragg case. Phys. Rev. Lett. 104, 165501 (2010).

16 Stadler, L.-M. et al. Hard x ray holographic diffraction imaging. Phys. Rev. Lett. 100, 245503 (2008).

17 Stickler, D. et al. Soft x-ray holographic microscopy. Appl. Phys. Lett. 96, 042501 (2010).

18 Zhang, F., Pedrini, G. \& Osten, W. Phase retrieval of arbitrary complex-valued fields through aperture-plane modulation. Phys. Rev. A 75, 043805 (2007).

19 Johnson, I. et al. Coherent diffractive imaging using phase front modifications. Phys. Rev. Lett. 100, $155503(2008)$. 
20 Quiney, H. M., Peele, A. G., Cai, Z., Paterson, D. \& Nugent, K. A. Diffractive imaging of highly focused x-ray fields. Nat Phys 2, 101-104 (2006).

21 Fienup, J. R. Reconstruction of a complex-valued object from the modulus of its Fourier transform using a support constraint. J. Opt. Soc. Am. A 4, 118-123 (1987).

22 Fienup, J. R. Phase retrieval algorithms: a comparison. Appl. Opt. 21, 2758-2769 (1982).

23 Marchesini, S. et al. X-ray image reconstruction from a diffraction pattern alone. $\underline{\text { Phys. Rev. B }}$ 68, 140101 (2003).

24 Higgins, G., Wolfe, R. The Relation of Definition to Sharpness and Resolving Power in a Photographic System. J. Opt. Soc. Am. 45, 121-129 (1955).

\section{ACKNOWLEDGEMENTS}

We thank Stefano Marchesini of LBNL for helpful discussions. This work at LBNL was supported by the Director, Office of Science, Office of Basic Energy Sciences, of the U.S. Department of Energy under Contract No. DE-AC02-05CH11231. Work in the group of S. D. K. at U. Oregon was supported by NSF through Grant No. DMR-0506241. 


\section{FIGURE LEGENDS}

Figure 1: Experimental geometry and microscopy of screen and test pattern.

(a) Experimental geometry to perform holography in reflection, showing the incident x-ray beam, the exit screen containing object and reference holes, and the charge-coupled device (CCD) detector. (b) Electron microscopy image of the exit mask. (c) Electron microscopy image of the pseudo-random test pattern.

Figure 2: Holographic data, Fourier transform, and phase-reconstructed image.

(a) Hologram data collected at the CCD, shown in log scale. (b) Fourier transform of the data in (a) gives the image in real space at the exit screen. The bright center is the autocorrelation while the side lobes are the cross-correlation term between object and reference hole. The two small lobes are the cross-correlation term between the two reference holes. (c) Image at the exit screen obtained after applying iterative phase retrieval method to the data. The big and small circle represents the structure of exit wave at the object and reference holes at the screen, respectively.

Figure 3: Back propagated images and comparison to SEM image. (a)-(d) Back-propagation by the given distance of the wave recovered (magnitude component) at the exit screen via iterative phasing algorithms discussed in the text. (e) The test pattern is shown from the perspective of the incident x-ray beam. Highlighted circular area shows the field of view of the incident x-ray. Excellent agreement is observed between (c) and (e). (f) Back-propagation of the cross-correlation term obtained directly from Fourier transform of the far field data.

Figure 4: Acutance and image quality metric.

Acutance (defined in the text) in the magnitude component of the wave at the exit screen after propagating in both forward and backward directions. The maximum after back propagating $97 \mu \mathrm{m}$ corresponds to the highest fidelity view of the test sample. 


\section{FIGURES}

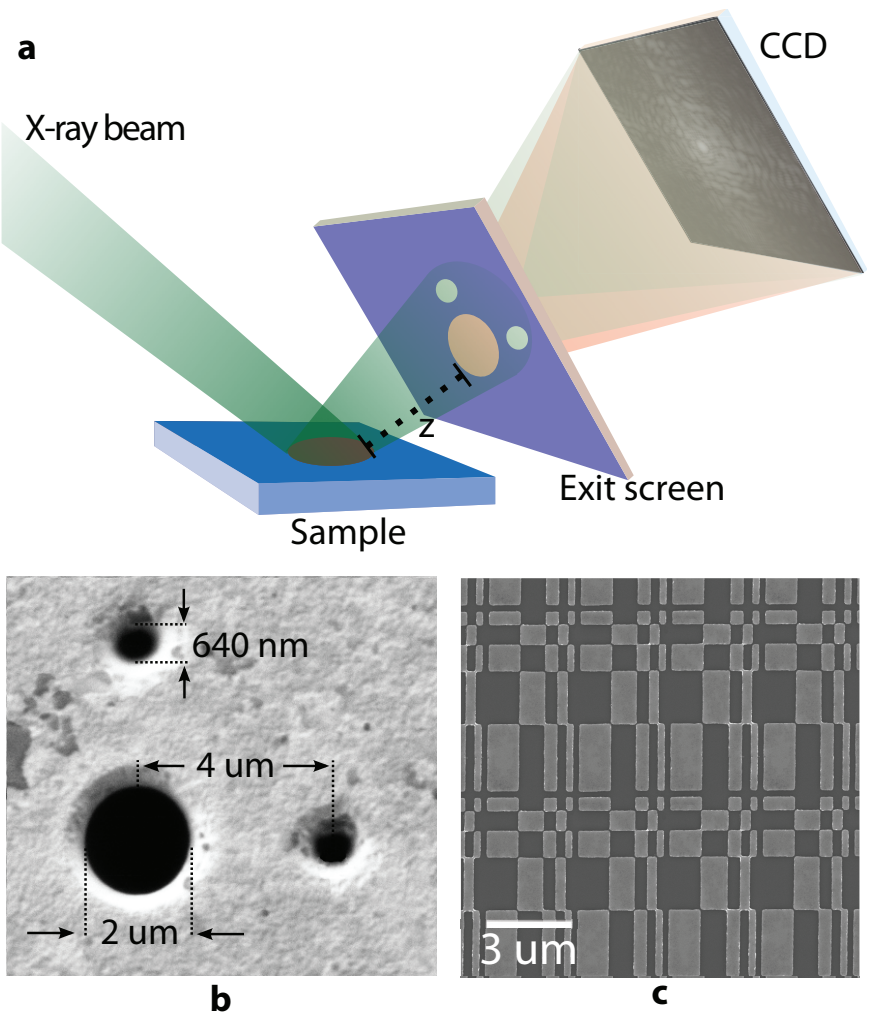

FIG. 1. 


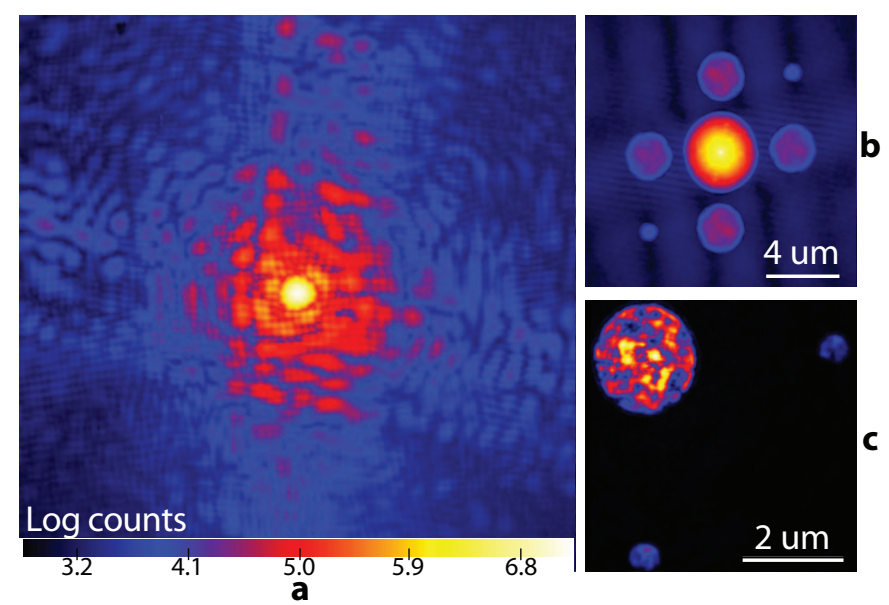

FIG. 2. 

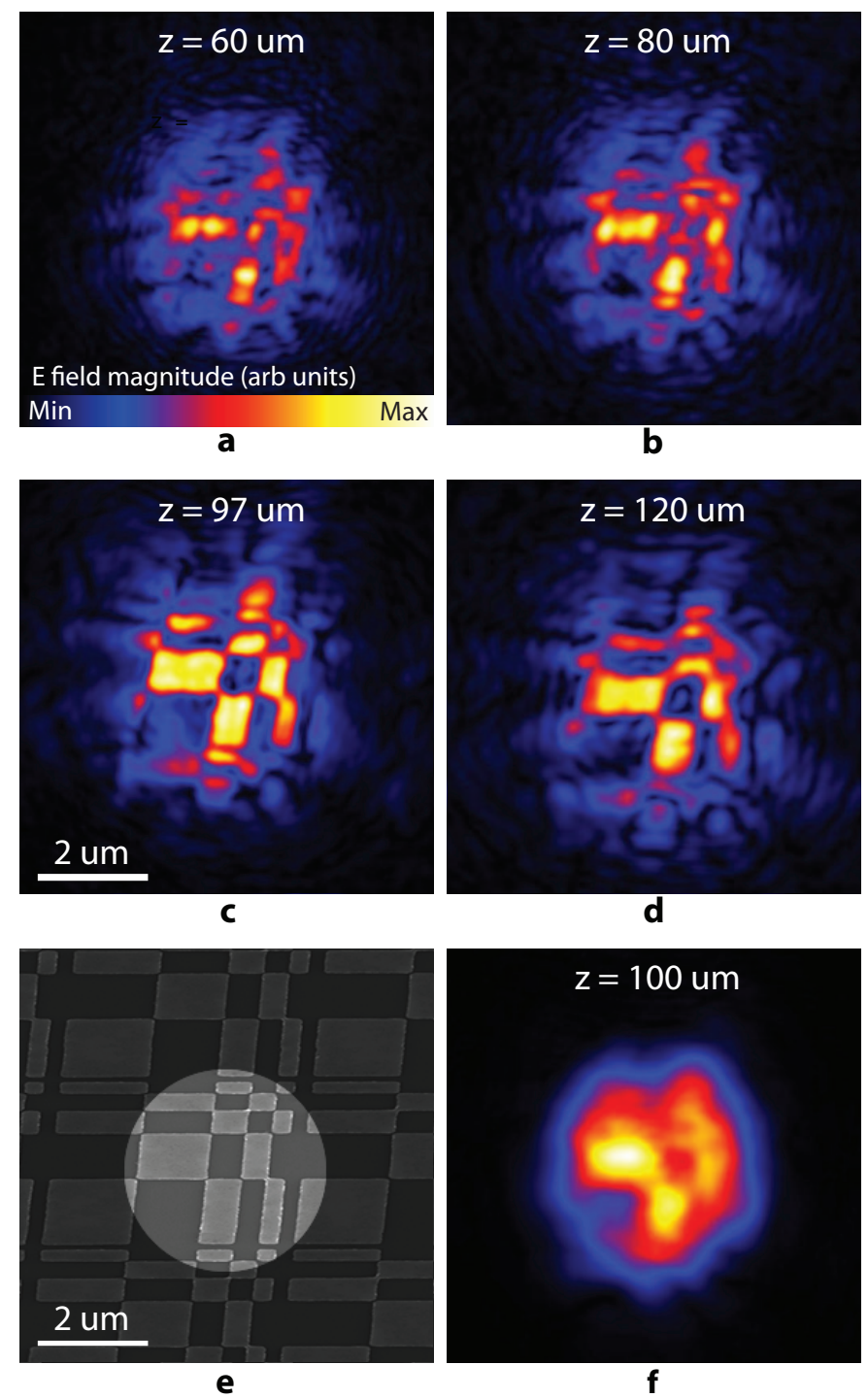

FIG. 3. 


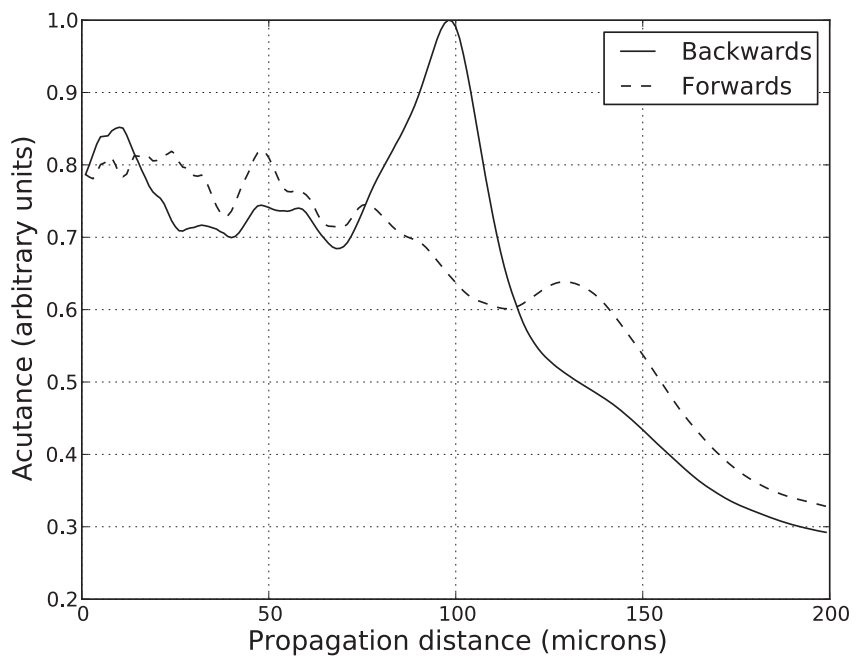

FIG. 4 